\title{
Poking the Future: When Should We Expect that Animal- Robot Interaction Becomes a Routine Method in the Study of Behavior?
}

\author{
Judit Abdai ${ }^{1 *}$ \& Ádám Miklósi ${ }^{1,2}$ \\ ${ }^{1}$ MTA-ELTE Comparative Ethology Research Group, Budapest, Hungary \\ ${ }^{2}$ Department of Ethology, Eötvös Loránd University, Budapest, Hungary \\ *Corresponding author (Email: abdai.judit@gmail.com) \\ Citation - Abdai' J., \& Miklósi, Á. (2018). Poking the future: When should we expect that animal-robot interaction \\ becomes a routine method in the study of behavior? Animal Behavior and Cognition, 5(4), 321-325. \\ https://doi.org/10.26451/abc.05.04.01.2018

\begin{abstract}
Animal-Robot Interaction (ARI) is not a novel approach in the study of animal behavior and cognition, but despite the methodological advances it provides, it is not as widespread as one may expect. The use of interactive, autonomous robots can give benefits which go well beyond the opportunities offered by early stimulus models. Such autonomous robots can help collecting data about animal behavior that is not possible achieve with any other methods. We hope that this collection of reviews and research studies may provide a new push to move this field forward.
\end{abstract}

Keywords - Animal-robot interaction, Ethorobotics, Social robotics, Behavior

In a review of animal-robot interaction (ARI) Krause, Winfield, and Deneubourg (2011, p 369) made a very important prediction: "Interactive robots have the potential to revolutionise the study of social behaviour because they provide several methodological advances." Thus, as editors of this volume, we thought nothing would be easier than to get a nice set of experimental papers from researchers worldwide. From the 1990's there was an increasing trend to build artificial agents to interact with various animal species; thus, the optimism of Krause et al. (2011) at that time seemed to be well-founded. The mechanical bee produced by Michelsen, Andersen, Storm, Kirchner, and Lindauer (1992) was able to communicate with forager bees in the hive. The robotic cockroach could be programmed to manipulate the spatial distribution of a group of living conspecifics (Halloy et al., 2007). Several types of robotic fish were engineered that could be successfully integrated in the swarms of living fishes (e.g., Aureli, Fiorilli, \& Porfiri, 2012; Bonnet, Kato, Halloy, \& Mondada, 2016; Butail, Polverino, Phamduy, Del Sette, \& Porfiri, 2014; Faria et al., 2010). Moreover, each of these innovations reached the headlines of the media, in parallel with achievements of robotics producing interactive social robots for human environments.

However, Krause et al.'s (2011) prediction has not been realized up to the present day. Apparently, the "revolution" has been postponed for a while. Despite the fact that there are year-by-year publications utilizing interactive robots to study animal behavior, this method has neither revolutionized the field, nor has it led to many novel insights. We think we do not harm anybody by honestly admitting it was very difficult to find a suitable number of papers to fill this volume. This collection of research articles should be seen as a new push to move the field forward, indeed to facilitate a "revolution" after which no going back is possible and the application of autonomous interactive robots becomes a standard 
tool both in the field and in the laboratory to investigate animal behavior from a new perspective. But as an introduction to this volume it may still be worthwhile to speculate why there is still limited interest for this approach in the animal behavior community.

\section{Is ARI "Only" a Method?}

At first sight, the interactive autonomous robots seem to be nothing more than complex and more sophisticated stimuli aimed to elicit animal behavior in an experimental setting. Ethologists are famous for coming up with simple models of conspecifics that helped to identify specific releasers of behavior. A piece of silver fish-shaped wood with a red underside turned out to be the best stimulus to elicit aggressive behavior in sticklebacks defending their territory (Tinbergen, 1948). A pair of appropriately sized black circles could be used to evoke begging behavior in young hatchlings (Tinbergen \& Perdeck, 1950). Obviously, replacing these early models with similar robotic agents arguably does not lead to new insights. However, robotic stimulation of animals could increase the objectivity of these experiments by replacing the human manipulation of these early makeshift models.

However, modern research using interactive autonomous robots can go well beyond studying single stimulus-behavior response coupling. The attack toward an intruder is only a very small part of the aggressive behavior pattern shown by a territorial stickleback male. Robotic models that are more similar and reactive to a living stickleback can help to reveal not just single reactions but also to discover more details of the organization of aggressive behavior including factors of winning or defeat. There is an increasing trend to investigate behavior not only as a compilation of frequencies or durations of action but rather as a functioning system; a network of interacting components that produces predictable outcomes of behavioral actions depending on a range of contextual factors. Ethological models of complex behaviors described by, for example, Baerends (1985) or Archer (1976) could be developed to a more advanced, realistic level by the means of interactive autonomous robots. Thus, although these robots may be seen merely as more sophisticated tools, in reality, their application can greatly advance modelling behavior.

\section{Technology and Researchers of Animal Behavior}

Ethologists and other researchers captivated by animal behavior are typically not inclined to employ technology to interact with their subjects of study. The tradition, which attracts people to this field, is the excitement generated by the knowledge that, by watching animals, one can get insights into their survival, evolution or mind. While observation of natural or naturalistic behavior is the principle of ethology, in recent years, more controlled experimental methodologies have been developed. In parallel, there is a trend to rely on statistically meaningful sample sizes in these studies, and large amounts of data collection are now commonplace, not only in laboratory settings but also in the case of field research. Cameras and laptops are now used overwhelmingly, and complex statistical calculations are applied in data evaluation. Thus, technology has already infiltrated the study of animal behavior. It may seem that the deployment of robots is only an inch away, but we have to be brave to take this last step.

\section{Robot Technology is Complex and Somewhat Unreliable}

It is important to note that, in the early research on animal-robot interaction, the enthusiasm of the engineers played probably a much larger role than the interest of ethologists. For the younger generation of researchers, it is difficult to imagine that computers were also not too reliable 30 to 35 years ago, and despite much technical effort, present day interactive robots are also in that state. In practice, this means that there are no off-shelf robots to work with, rather - researchers in animal behavior need to work closely together with robotic engineers not only during the planning and preparation of these agents but also during the entire experimental procedure. Although, at first sight, this may not sound very 
encouraging, collaboration between roboticists and ethologists could be very fruitful not only for technical reasons but also for the advance of science.

\section{Biorobotics, Ethorobotics and Animal/Human-Robot/Computer Interaction}

There is a plethora of approaches in robotics that have their own definitions and their own specific proponents, so it is not easy for newcomers to know their way around. All of these specific subfields have their own goals but eventually, there is a need for an overarching discipline. The main aim of biorobotics is to build agents that eventually mimic some aspects of living beings (Webb, 2000). This could mean moving with four legs (Chernova \& Veloso, 2004), or climbing like a gecko (Kim et al., 2008), or a hardware that simulates the escape system of a cockroach (Mongeau et al., 2012). In contrast, ethorobotics aims to design artificial agents with specific function by using insights of behavior without any constrains to mimic a particular living species (Miklósi, Korondi, Matellán, \& Gácsi, 2017). Such interactive robots may combine dog and human behavior skills to achieve their target function (e.g., Gergely, Petró, Topál, \& Miklosi, 2013; Miklósi \& Gácsi, 2012). The "interactive" robotic approaches focus on social behavior, and reflect, at the moment, a truly descriptive approach, and for such practical reasons it is less important whether we refer to 'animals' (human or nonhuman) or we call these agents "robots" or "computers", although ultimately the robot seems to be a more general, inclusive term.

We envisage that the use of such dynamic agents in animal behavior will eventually produce highly skilled robots that can, in various ways, engage in interactions with humans and nonhumans, and, in parallel, this will also increase our understanding how the minds of living animals work, which in turn will facilitate building better agents. Once this positive feedback starts to kick in, the "revolution" in ARI as envisaged by Krause et al. (2011) will be inevitable.

\section{Technology Improves over Time and is Expensive}

The last hindrance in this field is that researches have to get used to the fact that robotics is changing rapidly, and it is also expensive in comparison to the everyday costs of running experiments in animal behavior. Being a pioneer, building or getting built the first prototype for a specific interactive agent may cost much time and money. However, both engineers and ethologists can gain a lot of experience during this time and approaching the problem step by step is also useful.

There are also short-term replacements for autonomous agents. In our laboratory we have used remote controlled toy cars (renamed as unidentified moving object, UMO) to study how dogs react to agents that show specific socially meaningful behavior toward them, and how differential experience affects dogs' behavior toward these objects (e.g., Abdai, Gergely, Petró, Topál, \& Miklósi, 2015; Gergely et al., 2015; Gergely, Compton, Newberry, \& Miklósi, 2016). Based on this experience, we have now a much better understanding of what kind of autonomous interactive robots should be built that have a higher potential to be regarded as social companions for the dogs.

\section{A Step Ahead: State of Animal-Robot Interaction}

In the present issue, we introduce different research areas in which robotic agents have been applied to study animal behavior and cognition.

Abdai, Korcsok, Korondi, and Miklósi (2018, this issue) provide a short guide for those who are entertaining the idea to study animal behavior by the means of robot technology. They introduce novel aspects of animal-robot interaction research that may be useful to apply in the future, in order to facilitate the development and transparency. These steps are important to broadcast the advantages of ARI in research in animal behavior and may facilitate the widespread use of this method.

Porfiri (2018, this issue) discusses how the application of the transfer entropy concept helps in detection and quantification of causal relationships in animal interactions. He validates the usefulness of this measurement by the means of zebrafish-robot interaction, and also outlines a wide range of 
possibilities to study group behavior. The robotic approach provides "controllable and customizable stimuli to generate a priori known cause-and-effect relationships." The review draws attention to a fundamental theoretical concept that can be used widely to investigate animal interactions, but also addresses advantages and limitations of this approach.

The emergence of prosocial behavior and evaluation of the others based on their prosocial or antisocial behavior is an important issue both in nonhuman and human species. Quinn et al. (2018, this issue) report that rats behave toward a robotic agent similarly as with a conspecific social partner ( $c f$. Abdai et al., 2015 in dog-robot interaction), but also discriminate between them based on their behavior. The results of this study provide a useful tool for future research in rat-robot interaction and may in comparative investigations on the topic as well.

Zamansky et al. (2018, this issue) studied canine anxiety by the means of dog-robot interaction and used a self-developed software tool for automatized motion analysis. In the not-too-distant future, such tools could be applied to objectively diagnose canine anxiety and may provide a basis for measurement of other disorders in dogs. Using automatic motion analyzers can provide large amount of data on animal behavior.

Hirskyj-Douglas and Read (2018, this issue) explored dog behavior using an interactive media device that allowed dogs not only to attend to media, but also gave them control over what was displayed. Results are important both with regard to dog behavior, and also to system design. Family companions living in human environment are exposed to technical advancements; thus, such research also informs animal welfare, either considering whether it has an effect on them, or investigating whether these devices can be used as tools for improving the welfare. The study of Hirskyj-Douglas and Read can further contribute to novel methodological approaches of investigations into animal behavior and cognition.

\section{Acknowledgements}

We would like to thank all authors their contribution to this special issue on animal-robot interaction, as well as the reviewers who volunteered their expertise to move this field forward. We would also like to thank the editors of Animal Behavior and Cognition for making the publication of this issue possible.

\section{References}

Abdai, J., Gergely, A., Petró, E., Topál, J., \& Miklósi, Á. (2015). An investigation on social representations: Inanimate agent can mislead dogs (Canis familiaris) in a food choice task. PLoS ONE, 10, e0134575.

Abdai, J., Korcsok, B., Korondi, P., \& Miklósi, A. (2018). Methodological challenges of the use of robots in ethological research. Animal Behavior and Cognition, 5, 326-340. doi: https://doi.org/10.26451/abc.05.04.02.2018

Archer, J. (1976). The organisation of aggression and fear in vertebrates. In P. P. G. Bateson \& P. H. Klopfer (Eds.), Perspectives in ethology (Vol. 2, pp. 231-298). Boston, MA: Springer.

Aureli, M., Fiorilli, F., \& Porfiri, M. (2012). Portraits of self-organization in fish schools interacting with robots. Physica D, 241, 908-920.

Baerends, G. P. (1985). Do the dummy experiments with sticklebacks support the IRM-concept? Behaviour, 93, 258-277.

Bonnet, F., Kato, Y., Halloy, J., \& Mondada, F. (2016). Infiltrating the zebrafish swarm: Design, implementation and experimental tests of a miniature robotic fish lure for fish-robot interaction studies. Artificial Life and Robotics, 21, 239-246.

Butail, S., Polverino, G., Phamduy, P., Del Sette, F., \& Porfiri, M. (2014). Fish-robot interactions in a freeswimming environment: Effects of speed and configuration of robots on live fish. In A. Lakhtakia (Ed.), Proceedings of SPIE, Bioinspiration, Biomimetics, and Bioreplication (Vol. 9055, p. 90550I). San Diego, CA: SPIE

Chernova, S., \& Veloso, M. (2004). An evolutionary approach to gait learning for four-legged robots. In IEEE/RSJ International Conference on Intelligent Robots and Systems (IROS) (Vol. 3, pp. 2562-2567). Sendai, Japan: IEEE. 
Faria, J. J., Dyer, J. R. G., Clément, R. O., Couzin, I. D., Holt, N., Ward, A. J. W., ...Krause, J. (2010). A novel method for investigating the collective behaviour of fish: Introducing "Robofish." Behavioral Ecology and Sociobiology, 64, 1211-1218.

Gergely, A., Abdai, J., Petró, E., Kosztolányi, A., Topál, J., \& Miklósi, Á. (2015). Dogs rapidly develop socially competent behaviour while interacting with a contingently responding self-propelled object. Animal Behaviour, 108, 137-144.

Gergely, A., Compton, A. B., Newberry, R. C., \& Miklósi, Á. (2016). Social interaction with an "Unidentified Moving Object" elicits A-not-B error in domestic dogs. PLoS ONE, 11, e0151600.

Gergely, A., Petró, E., Topál, J., \& Miklosi, A. (2013). What are you or who are you? The emergence of social interaction between dog and an Unidentified Moving Object (UMO). PLOS ONE, 8, e72727.

Halloy, J., Sempo, G., Caprari, G., Rivault, C., Asadpour, M., Tâche, F., ...Deneubourg, J. L. (2007). Social integration of robots into groups of cockroaches to control self-organized choices. Science, 318, 1155-1158.

Hirskyj-Douglas, I., \& Read, J. C. (2018). DoggyVision: Examining how dogs (Canis familiaris) interact with media using a dog-driven proximity tracker device. Animal Behavior and Cognition, 5, 388-405. https://doi.org/10.26451/abc.05.04.06.2018

Kim, S., Spenko, M., Trujillo, S., Heyneman, B., Santos, D., \& Cutkosky, M. R. (2008). Smooth vertical surface climbing with directional adhesion. IEEE Transactions on Robotics, 24, 65-74.

Krause, J., Winfield, A. F. T., \& Deneubourg, J. L. (2011). Interactive robots in experimental biology. Trends in Ecology and Evolution, 26, 369-375.

Michelsen, A., Andersen, B. B., Storm, J., Kirchner, W. H., \& Lindauer, M. (1992). How honeybees perceive communication dances, studied by means of a mechanical model. Behavioral Ecology and Sociobiology, 30, $143-150$.

Miklósi, Á., \& Gácsi, M. (2012). On the utilization of social animals as a model for social robotics. Frontiers in Psychology, 3, 75.

Miklósi, Á., Korondi, P., Matellán, V., \& Gácsi, M. (2017). Ethorobotics: A new approach to human-robot relationship. Frontiers in Psychology, 8, 958.

Mongeau, J. M., McRae, B., Jusufi, A., Birkmeyer, P., Hoover, A. M., Fearing, R., \& Full, R. J. (2012). Rapid inversion: Running animals and robots swing like a pendulum under ledges. PLOS ONE, 7, e38003.

Porfiri, M. (2018). Inferring causal relationships in zebrafish-robot interactions through transfer entropy: A small lure to catch a big fish. Animal Behavior and Cognition, 5, 341-367. https://doi.org/10.26451/abc.05.04.03.2018

Quinn, L.K., Schuster, L.P., Aguilar-Rivera, M., Arnold, J., Ball, D., Gygi, E., Heath, S., Holt, J., Lee, D.J., Taufatofua, J., Wiles, J., \& Chiba, A.A. (2018). When rats rescue robots. Animal Behavior and Cognition, 5, 368-379. https://doi.org/10.26451/abc.05.04.04.2018

Tinbergen, N. (1948). Social releasers and the experimental method required for their study. The Wilson Bulletin, $60,6-51$.

Tinbergen, N., \& Perdeck, A. C. (1950). On the stimulus situation releasing the begging response in the newly hatched herring gull chick (Larus argentatus argentatus Pont.). Behaviour, 3, 1-39.

Webb, B. (2000). What does robotics offer animal behaviour? Animal Behaviour, 60, 545-558.

Zamansky, A., Bleuer-Elsner, S., Masson, S., Amir, S., Magen, O., \& van der Linden, D. (2018). Effects of anxiety on canine movement in dog-robot interactions. Animal Behavior and Cognition, 5, 380-387.

https://doi.org/10.26451/abc.05.04.05.2018 